\title{
Minimization of Spectrum Fragmentation for Improvement of the Quality of Service in Multifiber Elastic Optical Networks
}

\author{
Boris Stephane ZOUNEME ${ }^{1}$, Georges Nogbou $\mathrm{ANOH}^{2}$, Souleymane OUMTANAGA ${ }^{3}$ \\ Institut National Polytechnique Félix Houphouët-Boigny, Yamoussoukro, Côte d'Ivoire ${ }^{1,3}$ \\ Université Virtuelle de Côte d'Ivoire, Abidjan, Côte d'Ivoire ${ }^{2}$ \\ Laboratoire de Recherche en Informatique et Télécommunication, Abidjan, Côte d'Ivoire ${ }^{1,2,3}$
}

\begin{abstract}
Internet data traffic is still growing considerably in recent decades. In view of this exponential and dynamic growth, elastic optical networks are emerging as a promising solution for today's flexibly allocated bandwidth transmission technologies. The setup and release of dynamic connections with different spectrum bandwidths and data rates leads over time to spectrum fragmentation in the network. However, single-fiber eastic optical networks are faced with the problem of optical spectrum fragmentation. Spectrum fragmentation refers to small blocks, isolated, non-aligned spectrum segments which is a critical issue for elastic optical network researchers. With the advent of multifiber, this fragmentation ratio has become more pronounced, resulting in a high blocking ratio in multifiber elastic optical networks. In this paper, we propose a new routing and spectrum allocation algorithm to minimize fragmentation in multifiber elastic optical networks. In the first step, we define different virtual topologies as many as there is fiber, for each virtual topology, the $k$ shortest paths are determined to find the candidate paths between the source and the destination according to the minimization of a proposed parameter called allocation cost. In the second step, we apply the resource allocation algorithm followed by the choice of the optimal path with a minimum energy cost. Blocking probability and spectrum utilization are used to evaluate the performance of our algorithm. Simulation results show the effectiveness of our proposed approach and algorithm.
\end{abstract}

Keywords—Routing and spectrum allocation; fragmentation; multifiber elastic network; quality of service

\section{INTRODUCTION}

The remarkable development of wireless communication, especially 5G and services such as IPTV, Big Data, Cloud Computing, distance learning, IOTs, etc., introduces significant challenges in transmission technologies and systems for routing and switching large volumes of data, as well as network costs and energy consumption. According to Cisco VNI 2017, consumer Internet traffic is expected to increase by $27 \%$ between 2016 and 2021 [1][2], requiring a high-capacity network infrastructure with optical resources. Wavelength Division Multiplexing (WDM) technology has been proposed to support this exponential traffic growth. However, in WDM networks, due to the fixed $50 \mathrm{Ghz}$ grid and the rigidity of resource allocation, it leads to abusive and inefficient use of bandwidth. Elastic Optical Networks were then proposed to support variable bandwidth allocation using O-FDM (Optical
Frequency Division Multiplexing) technology according to demand with a more flexible spectral grid. O-OFDM allows continuous optical carriers and variable numbers of subcarriers on a channel. In elastic optical networks based on OFDM, the optical spectrum is divided into a number of frequency slot intervals with a width of 6.25 or $12.5 \mathrm{GHz}$ [3][4].

Several themes related to this new high-speed network technology are of interest for in-depth research. Continuity and contiguity constraints in the routing process and resource allocation (optical paths and spectrum) in single-fiber elastic optical networks; as well as the continuous establishment and release of optical connections with different spectrum bandwidths and data rates lead over time to spectrum fragmentation which is a crucial problem for elastic optical network research specialists. This spectrum fragmentation refers to small blocks of small, isolated, non-aligned spectrum segments with serious consequences, as it can lead to misuse of bandwidth and a high probability of blocking. Several routing and spectrum allocation algorithms have been proposed in monofiber. However, with the advent of multifiber, which provides greater flexibility in the allocation of spectrum slots in elastic optical networks, this fragmentation has been accentuated, leading to a high blocking probability and a lower QoS in the network. In this paper, we propose a new routing and spectrum allocation solution that significantly improves the state of multifiber elastic optical networks by taking into account power saving and a proposed parameter called allocation cost in the choice of the optimal path.

The rest of this paper is organized as follows. Section II introduces the architecture of elastic optical gratings and defines the problem. Section III presents the mathematical modelling of the multifiber elastic optical grating. Section IV describes the spectrum management strategy and the routing and spectrum allocation algorithm taking into account power consumption. The results of the performance evaluation of our routing and spectrum allocation strategy are presented in section V. Section VI concludes this paper.

\section{RELATED WORK}

Optical elastic networks are varied, high-speed data transport networks that require fairly large resources. This new type of optical transport network technique is based on optical modulation OFDM (Orthogonal Frequency Division 
Multiplexing) [5][6]in which the frequency grids are flexible from one connection request to another, unlike the fixed frequency grids of conventional WDM optical networks.

Through its flexibility, elastic optical network (EON) offers new features in terms of segmentation and aggregation of spectral resources (subwavelengths and super-wavelengths), efficient adaptation of multiple data rates, as well as elastic variation of the allocated resources. OFDM technology allows the channels of neighbouring subcarriers to overlap, as shown in Fig. 1.

This increases the spectral efficiency of the transmission. For this purpose, an optical signal is produced by a variable bandwidth OFDM transponder using the technology with just the right spectral resources to meet the customer's demand. This technique makes it possible to provide spectrum frequencies according to the needs of traffic from source to destination. Advances in optical transmission techniques and devices have led to the emergence of elastic optical networks. The introduction of advanced modulation formats and Wavelength Cross-Connects (WXC) optical cross-connects (WXCs) allows the increasing volume of traffic to be carried over long distances without opto-electronic-to-optical (OEO) conversion. Paths with bandwidths determined by the customer's traffic volume are allocated by flexible ratio transponders from the transmitter and are sent via Wavelength Cross-Connects (WXC) with variable bandwidth (BandwithVariable BVT) to the receiver as shown in Fig. 2.

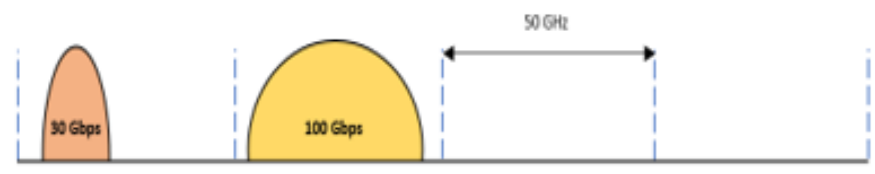

(a) Fixed grid

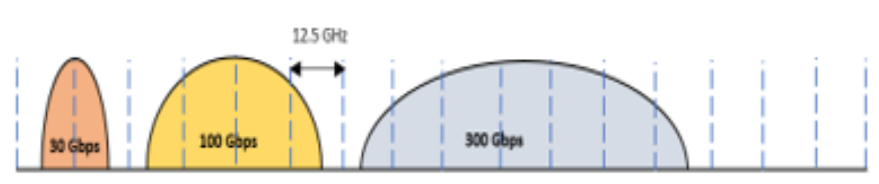

(b) Flexible grid

Fig. 1. Fixed Frequency Grid/Flexible Frequency Grid.

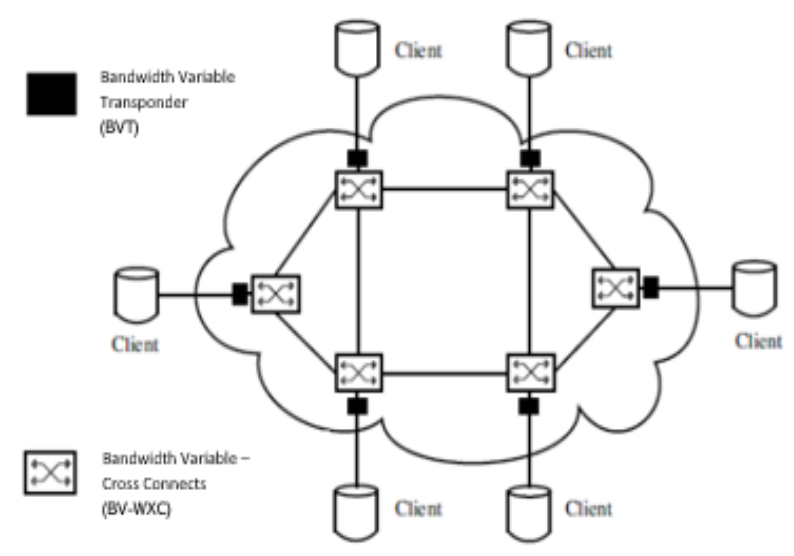

Fig. 2. Architecture of Elastic Optical Networks.
The system is based on Orthogonal Optical Frequency Division Multiplexing (OFDM). A frequency-locked multicarrier generator is used to generate equally spaced subcarriers.

The generated subcarriers are first separated by a wavelength division demultiplexer (DMUX), then individually modulated with parallel modulators, and finally coupled to generate a superimposed spectral superchannel. There are three transponder models, namely the Mixed Line Ratio (MLR) model, the Multi Flow (MF) model and the Bandwidth Variable (BV) model. The MLR model uses a few types of transponders, each with a different bit ratio, e.g. 40, 100 and $400 \mathrm{~Gb} / \mathrm{s}$ transponders to meet a wide range of traffic demands [7].

The MF model uses an MF transponder with several subtransmitter-receivers, which can be allocated to different traffic requests, each of which has a fixed throughput capacity. The BV model supports all types of traffic requests with a single BV transponder, which allocates as few spectrum resources as possible to traffic requests with a maximum bit ratio of $400 \mathrm{Gbps}$. As shown in the study presented in [7], the $\mathrm{BV}$ model provides better spectrum and the lowest consume ratio. It uses different modulation formats such as 16-QAM, 64-QAM and QPSK (Quadrature Phase Shift Keying). In addition, it offers a better balance between spectral efficiency and transmission range. Due to the reduction of active resources, a new generation of optical transponders (S-BVT) has been investigated in [8]. Flexible grating optical networks allow efficient use of spectrum resources by using the 12.5 $\mathrm{GHz}$ frequency instead of the traditional fixed $50 \mathrm{GHz}$ spacing, however introducing spectrum fragmentation (FS).

In the literature, SF is considered a crucial and major problem in EON, especially in a context of dynamic traffic. In this work, we present a comprehensive analysis and an accurate assessment of spectrum fragmentation in elastic optical networks.

The problem of fragmentation already exists in areas other than optical networks. It has been addressed for the management of synchronous optical networks (SONET) and code division multiple access (CDMA) systems in wireless networks [9] [10]. Data fragmentation has been studied for some context of EON (routing, number of locations, spectrum continuity constraint, network size, etc.) makes the MSDS issue different. MSDS in optical networks can be approached in two dimensions: horizontal fragmentation and vertical fragmentation as shown in Fig. 3.

In EON, connection requests are dynamically allocated according to continuity and contiguity constraints [11][12]. The continuity constraint must guarantee the use of the same wavelength or spectrum slot from end to end of an optical connection and leads to so-called horizontal fragmentation.

However, in flexible optical networks, lightpaths may be established without taking into account the spectrum continuity constraint. In this case, wavelength converters are required. However, service providers want to avoid the use of wavelength converters because of their additional deployment costs. 


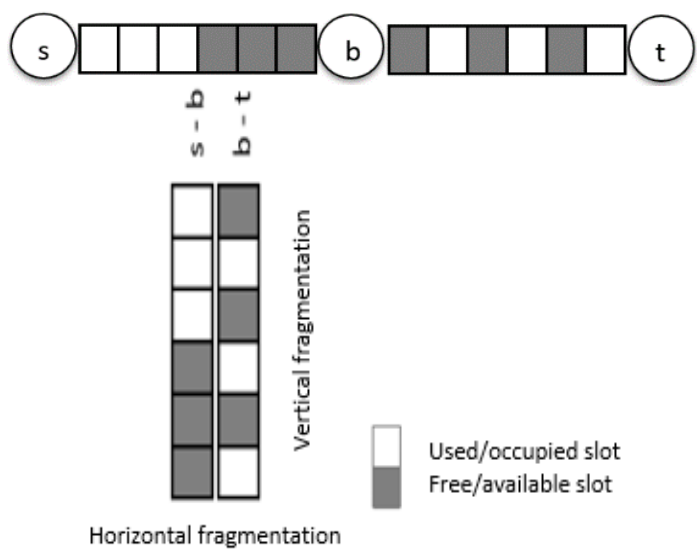

Fig. 3. Example of Vertical and Horizontal Fragmentation, s-b and b-t links are Horizontally Fragmented and Available/Free Slots are Vertically Fragmented.

It is clear that all these facts inevitably lead to the fragmentation of the optical spectrum which is a serious problem in ROE which is more accentuated in the dynamic context of the network in which requests arrive and disappear randomly. In such networks, new requests are therefore more likely to be blocked due to fragmentation [13]. Poor management of resource allocation significantly increases the ratio of fragmentation both in the path, in the link and in the network as a whole, leading to a deterioration of spectrum usage and huge losses on the operator side [14]. Several methods have been proposed to solve the problem of spectrum fragmentation, the simplest method is a fragmentation-aware Routing and Spectrum Allocation (RSA) algorithm that specifies how traffic requests are routed to minimize fragmentation [15]. Approaches to divide blocked demand into sub-demands in order to use finer granularities have also been proposed [16]. Another intuitive method is to defragment the spectrum so that free blocks are contiguous [17]. These methods are either complex, inefficient or difficult to implement. A new spectrum allocation strategy and a path selection scheme based on both the new network state after demand establishment and the probability of path selection have been proposed to eliminate fragmentation in multifiber elastic optical networks [18]. A quantitative parameter for evaluating link fragmentation is proposed to eliminate fragmentation by supporting a larger number of connections [19].

\section{Mathematical Modeling OF THE MultifiBeR ELASTIC OPTICAL NETWORK}

The topology of our network is defined by the graph

$\mathrm{G}(\mathrm{E}, \mathrm{L}, \mathrm{SF}, \mathrm{D})$ where :

- E represents all the nodes of the network by a set of optical distribution frames (OXCs).

- $\mathrm{L}=\{(\mathrm{i}, \mathrm{j})$ with $\mathrm{i} \neq \mathrm{j}\}$ represents the set of links in the network where each link contains several fibers assuming that the number of fibers on each link is identical and $\mathrm{i}$ and $\mathrm{j}$ represent the vertices of the graph.
- $S F=\left\{f_{s_{1}}^{l}, f_{s_{2}}^{l}, \ldots, f_{s_{n}}^{l}\right\}$ with $n \in \mathbb{N}$ such as $1 \leq l \leq \mathrm{F}$ and $\mathrm{n} \geq 1$ where $\mathrm{F}$ is the number of fiber per link and $\mathrm{n}$ is the number of slots.

- $\mathrm{D}=\left\{d_{1}, d_{2}, \ldots, d_{n}\right\}$ represents the set of network connection requests.

A connection request $c r$ between a source $s$ and a destination $d$ with a ratio $\omega(\mathrm{Gb} / \mathrm{s})$ is represented by:

$c r=\left(s_{c r}, d_{c r}, \omega_{c r}\right)$

The number of frequency slots NSF for a connection request $c r$ on the path $p$ is calculated according to the following expression in [2]:

$\operatorname{NFS}(p(c r))=\frac{\omega_{c r}}{\mathrm{M}(\mathrm{p}(\mathrm{cr})) \cdot \omega_{s}}+g b$

where gb represents the guard band

- $\mathrm{M}(p(c r))$ is a function that returns the coefficient of the appropriate modulation format:

$M\left(p(c r)=\operatorname{Modform}\left(\sum_{(i, j) \in p} l(i, j)\right)\right.$

- $\omega_{s}$ is the optical signal speed for a frequency slot

where $l$ is the length of the link $(\mathrm{i}, \mathrm{j})$ on the path $p$.

In the case of adaptive routing, the capacity of a slot and the range of the optical signal depends on the modulation format chosen for a connection. In addition, the use of transponders at different linear rates based on the optical technique of orthogonal frequency division multiplexing without dispersion (CO-OFDM) in EON can exploit several modulation formats for different subcarriers and provide flexibility and heterogeneous handling of connection requests.

Therefore, the transmission ratio for a subcarrier may vary depending on the modulation format, namely BPSK, QPSK, 4QAM, 16-QAM, 32-QAM and 64-QAM.

The table I is a summary of the energy consumed by a slot according to the modulation format.

TABLE I. ENERGY CONSOMPTION A SLOT IN FUNCTION OF MODULATION FORMAT AND OPTICAL RANGE

\begin{tabular}{|l|l|l|l|}
\hline $\begin{array}{l}\text { Optical } \\
\text { modulation } \\
\text { format }\end{array}$ & $\begin{array}{l}\text { Slot capacity } \\
(\mathrm{Gb} / \mathrm{s})\end{array}$ & $\begin{array}{l}\text { Energy consumed by } \\
\text { a transponder }(\mathrm{w})\end{array}$ & $\begin{array}{l}\text { Reach } \\
(\mathrm{km})\end{array}$ \\
\hline BPSK M=1 & 12.5 & 112.374 & 4000 \\
\hline QPSK M=2 & 25 & 133.416 & 2000 \\
\hline 8-QAM M=3 & 37.5 & 154.457 & 1000 \\
\hline 16-QAM M=4 & 50 & 175.498 & 500 \\
\hline 32-QAM M=5 & 62.5 & 196.539 & 250 \\
\hline 64-QAM M=6 & 75 & 217.581 & 125 \\
\hline
\end{tabular}




\section{DynAmic Algorithm For Minimizing Fragmentation In MUltifiber Elastic OPTICAL NETWORKS}

In our approach of routing and allocation spectrum named AMF (Algorithm for Minimizing Fragmentation), we assume that a given connection request must be established on the same fiber between the source and the destination. Therefore, being in a multifiber context, we define as much virtual topology as there are fibers on a given link. When a $\mathrm{cr}$ connection request arrives in the network; several virtual topologies are created. For each virtual topology, we determine a physical path between the source node $s$ and the destination node $d$. Assuming the physical topology with two fibers per link $f_{1}$ and $f_{2}$ described by Fig. 4 . we will obtain two virtual topologies describe by Fig. 5 and Fig.6 respectively.

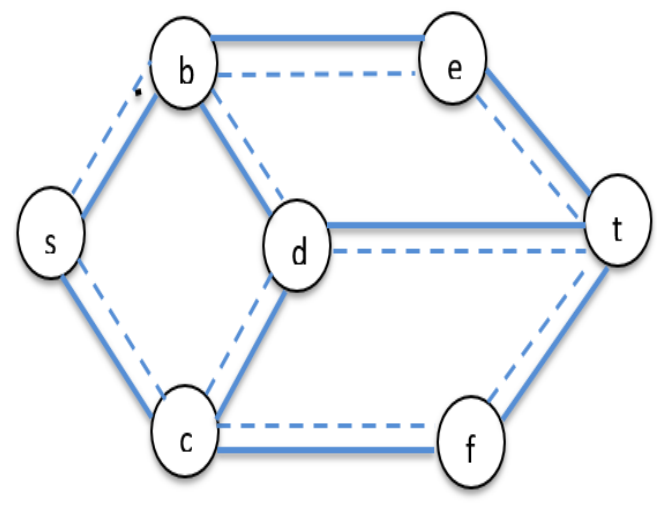

Fig. 4. Multifiber Network.

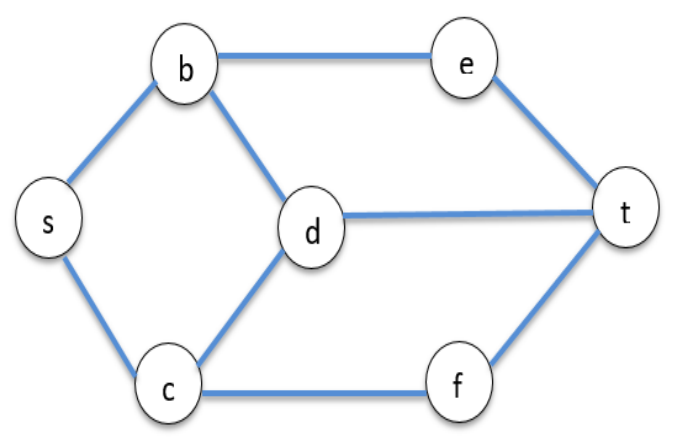

Fig. 5. Virtual topology 1 from $\mathrm{f}_{1}$.

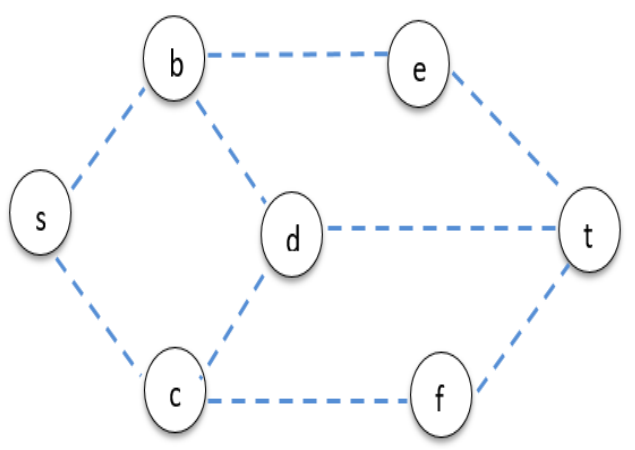

Fig. 6. Virtual Topology 2 from $\mathrm{f}_{2}$.
An optical path is characterized by source, destination and optical fiber as follows:

$p\left(\mathrm{~s}, \mathrm{~d}, \mathrm{f}_{\mathrm{i}}\right)$ where $s$ is the source, $d$ is the destination and $i$ represents the fiber.

After the representation of the different virtual topologies, for each virtual topology, we calculate the shorter paths from $\mathrm{s}$ to $\mathrm{d}$. For each path found, we calculate the number of frequency slots needed to establish the connection from (2). From the resources available on the path, we determine whether this path has the resources for the establishment of the connection request. If so, we try to determine the lightpaths (physical paths + spectral resources) which are eligible. It is then assumed that for each of the $\mathrm{N}$ topologies and for each of the shorter paths from source to destination, resources are determined for each physical path.

We assume that for $\mathrm{k}$ physical paths, we obtain $k^{\prime}$ optical paths with $k^{\prime} \leq k$ where $k$ is a natural number for each virtual topology. We will have at most $N^{\prime} * k^{\prime}$ eligible optical paths for the network; where $N^{\prime} \leq N$.

However, the question related to this approach that can be raised is which optical path will be chosen from the $N^{\prime} * k^{\prime}$ paths?

To answer this question, after determining the $N^{\prime} * k^{\prime}$ eligible optical paths; we are seeking to determine the fragmentation ratio.

In this case, the light path that will be chosen as the optimal path will be the one that consumes less energy and has the lowest fragmentation ratio, or if all light paths consume the same energy then the one with the lowest fragmentation ratio will be chosen. If this is not possible, the connection request is blocked.

\section{A. Fragmentation Parameter}

We can consider a fragmentation parameter that we will define as a criterion for choosing the best optimal path with the objective of minimizing fragmentation.

This fragmentation parameter, which we will name Allocation Cost AC, depends on the presence of the total number of frequency slots available on the path and the number of slot blocks.

This parameter is defined as follows:

$A C=\sum_{i=1}^{n} \exp \left(\frac{1}{s_{i}}\right)$

where, $S_{i}$ the number of frequency slots in block $i$ available on the path.

We illustrate this parameter as follows with three links $L_{1}$, $L_{2}$ eand $L_{3}$ for a connection request requiring three contiguous

frequency slots :

$L_{1}$

\begin{tabular}{|l|l|l|l|l|l|l|l|l|l|l|l|}
\hline 1 & 2 & 3 & 4 & 5 & 6 & 7 & 8 & 9 & 10 & 11 & 12 \\
\hline
\end{tabular}

$\mathrm{AC}\left(L_{l}\right)=4 e^{1}=10.873$ 
$L_{2}$

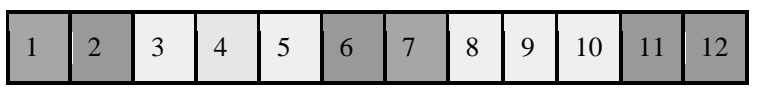

$\mathrm{AC}\left(L_{2}\right)=e^{\frac{1}{3}}=1.395$

$L_{3}$

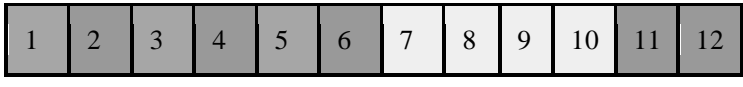

$\mathrm{AC}\left(L_{3}\right)=e^{\frac{1}{3}}=1.395$

We note that $\mathrm{AC}\left(L_{3}\right) \leq \mathrm{AC}\left(L_{2}\right) \leq \mathrm{AC}\left(L_{1}\right)$, when the cost of allocation is high this leads to the creation of small slot blocks with a low probability of being used for future connection requests and thus increases network fragmentation. So with regard to this fragmentation parameter when the value of this parameter is low the fragmentation of the link is reduced.

\section{B. Energy Cost of a Lightpath}

The energy cost of a lightpath for a $c r$ connection request is calculated according to the following parameters: the energy consumption of a slot, the energy consumption of an optical splitter along the link and the energy consumption of an Erbium Doped Fiber Amplifier (EDFA). Let the following expression be used:

$E C L(C(c r))=(\mathrm{NFS}(\mathrm{C}(\mathrm{cr}))-\mathrm{gb}) \times C_{\text {slot }} \times P C_{\text {slot }}+$ $\left(\sum_{(i, j) \epsilon C} C_{A m p} \times P C_{A m p} \times(\rho(i, j)+2)+\sum_{(i, j) \epsilon C} C_{O X C} \times\right.$

$\left.P C_{O X C}\right) \times \frac{N F S(C(c r))}{S U M_{\text {slot }}}$

with $\quad \rho(i, j)=\left\{\begin{array}{c}0, \text { if } \frac{l(i, j)}{d_{A m p}} \leq 1 \\ {\left[\frac{l(i, j)}{d_{A m p}}\right], \text { otherwise. }}\end{array}\right.$

The principle of determining physical paths by our ADCP algorithm, for each connection request that arrives on the network; we create as many virtual topologies as there are fibers on the network links defined by the $G$ graph. After creating the different topologies, we determine the shortest paths and for each path found, we determine the path that has resources available for the connection request.

If there is a path in the network that has resources available for the connection request, then the physical path and the spectral resources, i.e. the lightpath, are saved.

Then we calculate the energy cost for each path in order to determine the optimal path that will have the minimum energy cost.

Our method (AMF) selects the path and slots that use the minimum spectrum based on our AC cost of allocation parameter and a minimum energy cost, thus reducing bandwidth blocking for the overall network.

Network topology (G (E, L, SF, D))

Location of the OXC network nodes, flexible links, fibers and connection requests, the guard band are given as input.
The output will be the best lightpath with the lowest spectrum utilization and the lowest blocking probability.

For a given connection request and topology AMF algorithm will create the virtual topologies from the graph $(G$ (E, L, SF, D)), then for each virtual topology will determine the $\mathrm{k}$-shortest paths using ADCP algorithm and then find the available slots using the ADRS algorithm. If the available slots are found then the physical paths with available slots are saved. Then the best lightpath that has the minimum power cost and a low allocation cost is chosen according to ADMC algorithm to serve the connection request.

\section{Algorithm to detemine the physical path (ADCP)}

Input: Graph G

Output: Physical paths and resources

1: Create the virtual topologies from graph $\mathrm{G}$

2. For each connection request that arrives in the network

3: For each virtual topology

4: $\quad$ Determine the k-shortest paths

5: $\quad$ For each shortest path

6: $\quad$ Determine the resources for the connection request

7: $\quad$ If resource found then

8: $\quad$ Save the physical path and its resources

9: $\quad$ End if

10: $\quad$ End for

11: If lightpath found then

12: $\quad$ Choose the optimal path

13: Etablish the connection request

14: Otherwise

15: $\quad$ Go to step 3

16: $\quad$ End if

17: End for

18: End for

\section{Algorithm to Minimize Fragmentation (AMF)}

Input: Graph G

Output: Physical paths and resources

1: Create the virtual topologies from graph $\mathrm{G}$

2. For each connection request that arrives in the network

3: $\quad$ For each virtual topology

4: Determine the k-shortest paths using algorithm ADCP

5: $\quad$ For each shortest path

6: Determine the resources for the connection request using ADRS algorithm

7: $\quad$ If resource found then

8: $\quad$ Save the physical path and its resources

9: $\quad$ End if

10: $\quad$ End for

11: $\quad$ If lightpath found then

12: Choose the best optimal lightpath that has the minimum energy cost using algorithm ADMC

13: $\quad$ Save the optimal lightpath

14: $\quad$ End if

15: $\quad$ End for

16: If optimal lightpaths

17: Choose the best optimal lightpath that has the lowest

fragmentation ratio

18: End if

19: End for 


\section{Algorithm to determine spectral resource (ADRS)}

Input: Physical paths

Output: Resources to be used (free frequency slots)

1: Determine the number of slots for the connection request

2: Determine the blocks of slots for the path

3: Determine the free blocks of slots which are eligible for the connection

4: Determine the smallest eligible block among all the eligible blocks

5: Extract the resource blocks from this block for the connection request

In the principle of determining the best lightpath by our ADMC algorithm, for a given connection request, the lightpath with minimal energy cost is chosen. In the case where several lightpaths have the same minimum cost, the lightpath that reduces the fragmentation ratio is chosen.

\section{Algorithm to determine the best lightpath (ADMC)}

Input: All paths and the connection request

Output: Optimal lightpath for the connection request

1: Calculate the energy cost of each path

2: Determine the path with the minimum energy cost

3: If two paths have the same minimum energy cost then

4: $\quad$ Choose the path that reduces the fragmentation ratio

5: End if

\section{RESULTS}

\section{A. Assumptions}

In this section, we discuss the simulation results and evaluate the performance efficiency of our proposed AMF algorithm with the algorithm proposed in [20] in NSFNET and US-backbone topologies.

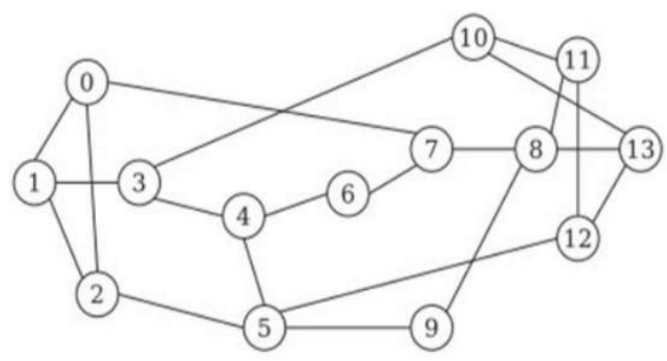

Fig. 7. NSFNET Topology.

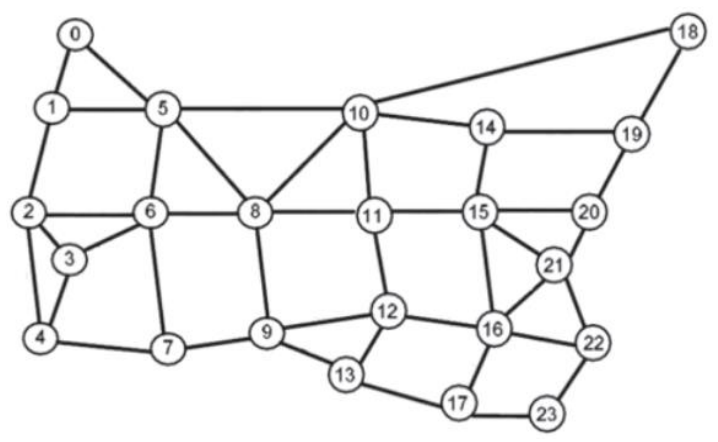

Fig. 8. US-Backbone Topology.
The NSFNET topology consists of 14 nodes and 22 links while the US-backone topology consists of 24 nodes and 43 links illustrated in Figs. 7 and 8. We assume that each link has an identical number of fibers $\mathrm{F}=10$. The total number of slots per fiber and the bandwidth of each slot are 352 and $12.5 \mathrm{Ghz}$ respectively.

Note also that in this paper the frequency conversion is not taken into account. Table II. shows the simulation parameters.

Between $10^{4}$ et $10^{5}$ connection requests arrive as dynamic traffic in the network following a sequential and random process. Each source-destination node pair is randomly selected. We estimate the capacity of a fiber link on the network at $4400 \mathrm{Gbps}$.

Thus, the performance of our algorithm is evaluated in terms of blocking probability and spectrum utilization ratio.

The blocking probability is defined as the ratio between the number of rejected connection requests and the total number of requests arriving on the network. Whereas the spectrum or spectrum resource usage ratio is the ratio of the number of occupied slots to the total number of available slots. For better network performance, the probability of blocking and spectrum usage rates should be as low as possible.

\section{B. Performance Evaluation}

Fig. 9 and 10 show the simulation results in terms of blocking probability in the NSFNET and US-backbone topologies respectively. Thus, we can observe that the curves in both figures follow roughly the same trend. The results of our AMF algorithm compared to those of the NSA algorithm show that the AMF strategy results in a significantly lower blocking probability for all traffic loads in both topologies.

TABLE II. SIMULATION PARAMETERS

\begin{tabular}{|l|l|}
\hline Parameter & Value \\
\hline Degree & 3 \\
\hline Number of slots/fiber & 352 \\
\hline Bandwidth of a slot & $12.5 \mathrm{Ghz}$ \\
\hline Capacity of a slot of $\mathrm{M}=1$ & $12.5 \mathrm{Gbps}$ \\
\hline Range debit & $12.5-400 \mathrm{Gbps}$ \\
\hline Capacity of a fiber link & $4400 \mathrm{Gbps}$ \\
\hline
\end{tabular}

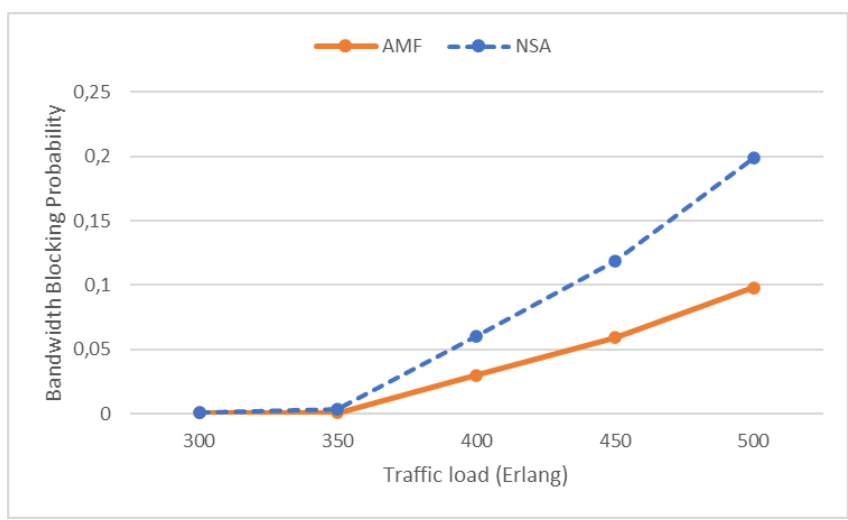

Fig. 9. Comparaison of Bandwidth Blocking Probability in NSFNET Topology. 


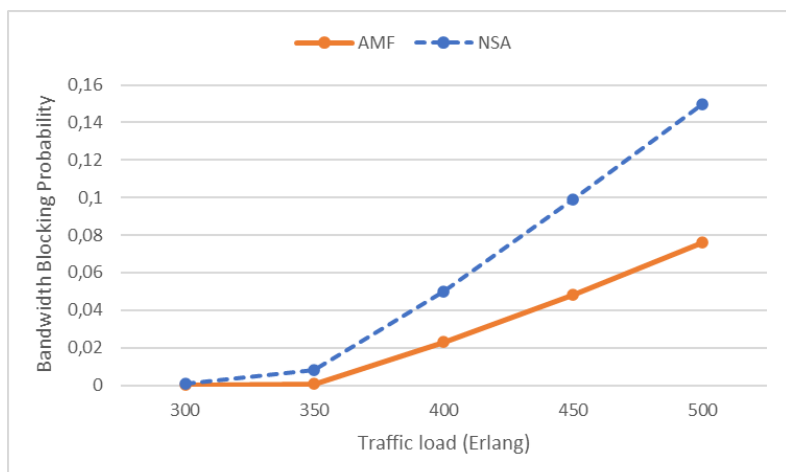

Fig. 10. Comparison of Bandwidth Blocking Probability in US-Backbone Topology.

Several observations can be made from the results obtained, namely: between 300 and 350 Erlang the probability of blogging is approximately the same for both approaches in both topologies. However, we find that a reduction of $16 \%$ and $19.4 \%$ is achieved in terms of blocking probability by our proposed AMF approach compared to the NSA approach for the US-backbone and NSFNET topologies respectively. This is due to the fact that as the unused spectrum becomes larger and larger, nodes reject incoming connection requests due to the limit of spectrum capacity. This shows the efficiency of both routing and spectrum allocation of our proposed algorithm.

The spectrum utilisation ratio as a function of network traffic load is shown in Figs. 11 and 12 for the NSFNET and US-backbone topologies. Note that the number of resources increases as the network load increases.

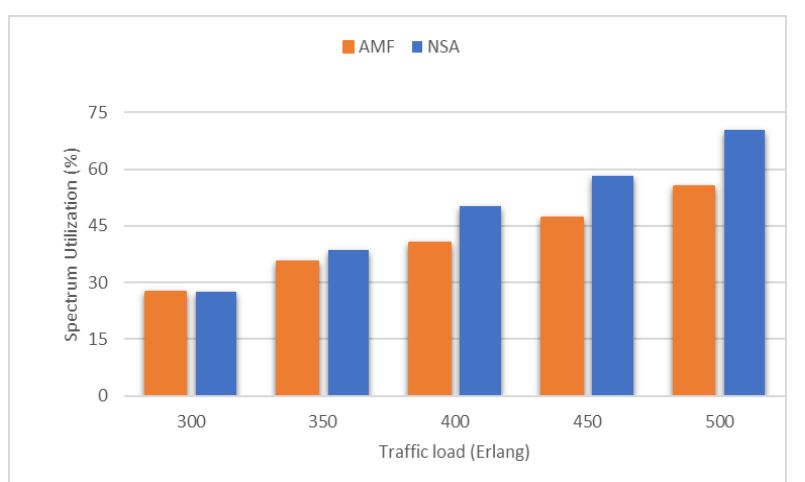

Fig. 11. Comparison of Spectrum utilization Ratio in NSFNET Topology.

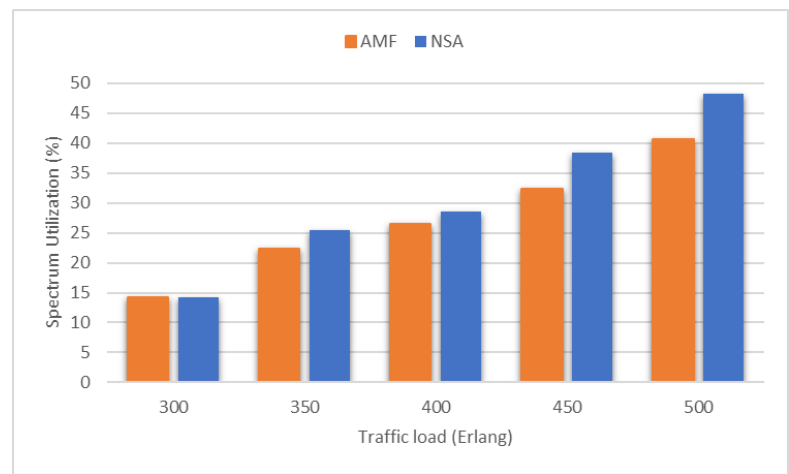

Fig. 12. Comparison of Spectrum utilization Ratio in US-Backbone Topology.
As we observe from these figures, our AMF approach requires a minimum ratio of use of spectrum resources. Simulation results show a gain of $18.7 \%$ and $38.9 \%$ in terms of spectrum usage efficiency for US-bacbone and NSFNET topologies respectively. It shows that the NSA algorithm has a slight impact on the quality of service which is of paramount importance for the profitability of the service provider and especially for the customer satisfaction. Hence the significant improvement of our AMF algorithm in terms of service quality. Note that taking into account the traffic load, [20] calculates the set of paths and the probability of path selection for each node in offline mode, which gives a better algorithmic complexity to our approach. However, in our AMF approach, the routing and spectrum allocation is done dynamically and allows to obtain a good performance in spectrum utilization ratio.

\section{CONCLUSION}

Routing and spectrum allocation is a major challenge in elastic optical networks. Dynamic and continuous establishment and withdrawal leads to fragmentation of the spectrum resulting in small, isolated and non-contiguous fragments, resulting in inefficient use of spectrum and increased probability of blocking. In this paper, we proposed a new algorithm to minimize fragmentation (AMF) with a new cost of allocation parameter (AC) for dynamic traffic demands in elastic optical networks with multiple fibers per link. Assuming that the connection is established on the same fiber between the source and the destination, we define as many virtual topologies as there are fibers on a given link taking into account minimum power consumption of network components in the choice of the optimal optical path. The performance of our proposed approach is evaluated by simulating NSFNET and US-backbone network topologies in terms of bandwith blocking probability and spectrum utilization efficiency to achieve better performance than existing algorithms in the literature.

In the future work, we plan to optimize bandwidth by increasing network capacity and reduce the complexity of network components by developing routing and spectrum allocation algorithms by integrating into multifiber elastic optical networks the Software Defined Optical Networks (SDONs) considered as next-generation networks with centralized controllers based on the Software Defined Network $(\mathrm{SDN})$.

\section{REFERENCES}

[1] Cisco, Cisco Visual Networking Index: Forecast and Methodology, 2016 - 2021. Cisco white paper, June 2017.

[2] T. Ahmed, S. Rahman, M. Tornatore, X.. Yu, K.. Kim, and B. Mukherjee, "Dynamic Routing and Spectrum Assignment in CoExisting Fixed/Flex-Grid Optical Networks", IEEE International Conference on Advanced Networks and Telecommunications Systems (ANTS), 2018.

[3] J. Wu, M. Xu, S. Subramaniam, and H. Hasegawa, "Routing, fiber, band, and spectrum assignment (RFBSA) for multigranular elastic optical networks" in IEEE Int. Conf. On Communications (ICC), 2017, pp. 1-6.

[4] J. Wu, S. Subramaniam and H. Hasegawa, (2015). " Comparison of OXC Node Architectures for WDM and Flex-Grid Optical Networks", 24th International Conference on Computer Communication and Networks (ICCCN), 2015. 
[5] N. G. Anoh., M. Babri, D. K. Ahmed, M. F. Roger, B. Aka and C Lishou, "An efficient hybrid protection scheme with shared/dedicated backup paths on elastic optical networks", Digital Communications and Networks, March 2017.

[6] C. C. Bijoy, N. Sarma, E. Oki, "Routing and Spectrum Allocation in Elastic Optical Networks: A Tutorial” IEEE Communication Surveys and Tutorial, vol.17, no.3, 2015.

[7] G. Zhang, M. D. Leenheer, A. Morea, B. Mukherjee", A survey on OFDM-based elastic core optical networking", IEEE Communications Surveys \& Tutorials, 2013.

[8] T. Tanaka, A. Hirano, and M. Jinno, "Impact of transponder architecture on the scalability of optical nodes in elastic optical networks," IEEE Communications Letters, vol. 17, 2013.

[9] M. S. Johnstone, P. R. Wilson "The memory fragmentation problem: solved?", In: Proceedings of the 1 st international symposium on Memory management, 1998.

[10] G. Bouyer. Les réseaux synchrones étendus PDH et SDH. Hermes, 1997.

[11] M. S. Johnstone, P. R. Wilson, "The memory fragmentation problem: solved?", In: Proceedings of the 1 st international symposium on Memory management (ISMM), 1998.

[12] J. Wu, M. Xu, S. Subramaniam, and H. Hasegawa, "Joint BandingNode Placement and Resource Allocation for Multigranular Elastic Optical Networks", Journal of Optical Communications and Networking, 10(8), C27, 2018.
[13] Y. Wang et al, "Spectrum consecutiveness-based routing and spectrum allocation in flexible bandwidth networks", Chinese Optics Letters, 2012.

[14] M. S. Johnstone, Wilson, P.R. "The memory fragmentation problem: solved?", In: Proceedings of the 1 st international symposium on Memory management, 1998.

[15] Y. Sone, A. Hirano, A. Kadohata, M. Jinno, O. Ishida," Routing and spectrum assignment algorithm maximizes spectrum utilization in optical networks", Optical Communication, 2011.

[16] A. Pages, J. Perello, S. Spadaro, J. Comellas, "Optimal route, spectrum, and modulation level assignment in split-spectrum enabled dynamic elastic optical networks", Opt. Commun. Netw. IEEE/OSA, 2014.

[17] R. Wang, , B. Mukherjee, "Provisioning in elastic optical networks with non-disruptive defragmentation", Lightwave Technol., 2013.

[18] W. Jingxin, S. Suresh and H. Hiroshi, "Dynamic Routing and Spectrum Assignment for Multi-fiber Elastic Optical Networks", Conference Paper, 2018.

[19] S. Krishan, S. Shree and S. Sujata, "Fragmentation Suppressed RSA Algorithm for Elastic Optical Network: A Quantitative approach", IIEEE British and Irish Conference on Optics and Photonics London, 2018.

[20] W. Jingxin, S. Suresh and H. Hiroshi, "Efficient Dynamic Routing and Spectrum Assignment for Multifiber Elastic Optical Networks", J. Opt Commun. Netw, vol. 11, 2019. 